\title{
EFFECTS OF SWEETENERS AND STORAGE ON THE ACIDITY, SOLUBLE SOLIDS AND SENSORIAL PROFILE OF LINGONBERRY JAMS
}

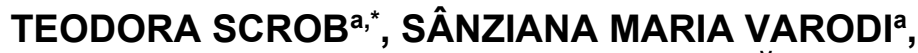 \\ GEORGIANA ALEXANDRA VINTILĂ ${ }^{a}$
}

\begin{abstract}
In this study, seven jam formulations were prepared, starting with the basic formulation, containing sucrose. This sweetener was replaced by fructose, erythritol, brown sugar, coconut sugar, stevia and saccharine, making these formulations a good alternative, some of them being also suitable for diabetic patients. Titratable acidity (TA) and total soluble solids (TSS) of lingonberry jams were evaluated for changes in jam quality during storage at $4^{\circ} \mathrm{C}, 25^{\circ} \mathrm{C}$ (under light conditions) and $25^{\circ} \mathrm{C}$ (under dark conditions) for 60 days. Moreover, a sensory evaluation was performed after 180 days of storage at $4^{\circ} \mathrm{C}$ to assess its consumer acceptance as compared to jam made with sucrose. During storage, TA and TSS increased in the case of all samples regardless of temperature conditions. ANOVA analysis of results revealed that the changes in TA and TSS were significantly affected by the type of sweetener used in jam formulation $(p<0.05)$. Jams formulated with coconut sugar and stevia were assessed by the sensory panel as the most acceptable.
\end{abstract}

Keywords: titratable acidity, total soluble solids, sucrose, sweeteners, sensory properties

\section{INTRODUCTION}

Due to the growing health concerns and higher incidence of obesity, metabolic syndrome and diabetes, during the recent decades there has been an increase in interest for low calorie food consumption [1,2]. These days' consumers are looking for high quality foods and there is considerable demand for fresh fruits and their products. Lingonberry fruit (Vaccinium vitis-

\footnotetext{
a Babeş-Bolyai University, Faculty of Chemistry and Chemical Engineering, 11 Arany Janos str., RO-400028, Cluj-Napoca, Romania,

*Corresponding author: teodora.scrob@ubbcluj.ro; teodora_scrob@yahoo.com
} 
idaea) is attaining popularity, as it is rich in bioactive compounds including anthocyanins, proanthocyanidins, flavonols or hydroxycinnamic acids [3] and also possesses various biochemical activities such as antioxidant and antibacterial effects [4]. However, these fruits are perishable and the lack of appropriate techniques for postharvest, transport and storage results in great losses. Fruit preservation techniques like candying, production of jams and fruit preserves extend significantly their shelf-life allowing their consumption all year [5]. In this way, jams are most preferred by consumers mainly due to their availability, sensory quality and low cost.

Sugar has a key technological role in the traditional jam's confection, as it influences the soluble solids content. Therefore, sugar plays an important role in physical, chemical, and sensorial properties of jams, also increasing its microbiological stability and hence safety [6]. However, large sugar consumption has been correlated with adverse effects on health, such as cardiovascular diseases, obesity and diabetes [7]. During the recent decades there has been an increase in interest for replacing sugar with other constituents. Fructose is the most commonly used sugar in jam formulations for people with diagnosed diabetes mellitus type 2 [8]. Sugar alcohols (erythritol, isomalt, lactitol, maltitol, mannitol, sorbitol), are attractive alternatives to sucrose as they are minimally metabolized (and thus we draw fewer calories from them), taste relatively similar to sucrose, and are naturally occurring [9]. Coconut sugar has become a popular alternative to white sugar due to its distinctive aroma, slightly acidic and smelling caramel, with a lower glycemic index than white sugar. Stevia is another natural sweetener, which is increasingly popular in the last years. It represents extract from the leaves of the plant Stevia rebaudiana (Bert.) which contains a high level of low-calorie sweetening compounds, known as steviol glycosides. Besides sweetening properties, Stevia rebaudiana extracts possess antioxidant, antimicrobial and antifungal activity $[10,11]$. Among synthetic sweeteners, saccharin is the oldest of this sweeteners category, with sweetness from 200 to 700 times more potent than sucrose. Synthetic sweeteners are in principle designed to mimic the sensory properties of sucrose, but in actuality usually exhibit additional taste notes other than sweetness, most frequently bitterness, or temporal characteristics that do not match that of sucrose [12]. This can lead to a lingering sweetness that consumers find off-putting [11].

Fruit jam incorporating alternative sweetener should have similar textural and rheological characteristics, as well as sensory properties to that of the traditional product [13]. Sugar-free jams are possible using alternative sweeteners also called low-calorie sweeteners, such as sorbitol, maltitol, and/or sweeteners like saccharin and cyclamate, responsible to cause 
weight gain [14]. However, the attainment of a suitable texture may be more difficult in sugar-free jams than in jams with other sweeteners. So the new product formulation has to result in similar texture, flavor, and other characteristics of the traditional product. Therefore, understanding of the factors that may influence the texture of processed foods is essential for the development of new products [5].

To our knowledge, the literature available at present is deficient in references about effects of different sweeteners on lingonberry jams properties during storage. Thus, the aim of this work was the development of lingonberry jam's formulations with different nutritional properties. For this purpose, the sucrose used in traditional jams was replaced by fructose, erythritol, brown sugar, coconut sugar, stevia and saccharine. The sensory properties of the products were evaluated in order to ascertain their acceptability. In addition, the changes in total soluble solids (TSS) and titratable acidity (TA) of lingonberry jams during storage at $4^{\circ} \mathrm{C}, 25^{\circ} \mathrm{C}$ (under light conditions) and $25^{\circ} \mathrm{C}$ (under dark conditions) for 60 days were monitored.

\section{RESULTS AND DISCUSSION}

\section{Effects of storage time and temperature on titratable acidity (TA) of jams samples}

Because acidity protects against the development of microorganisms, measuring TA is an important step in evaluating the quality of a jam. The TA values of jams samples obtained during 60 days of storage are presented in Table 1. TA increased during storage in the case of all samples regardless of temperature conditions. After 15 days of storage, maximum increase was observed in Jam 6 stored under light conditions (63.3\%), while the lowest increase was observed in Jam 3 stored at $4^{\circ} \mathrm{C}(4.16 \%)$. Storage at $4^{\circ} \mathrm{C}$ after 30 days determined an evident increase of TA in the case of Jam 2, from $7.69 \%$ to $34.5 \%$, respectively. After 60 days of storage, at $25^{\circ} \mathrm{C}$ (under light), in Jams 5 and 6 total acids increased to highest values of $0.364 \%$ and 0.385 $\%$, respectively. Storage at $4^{\circ} \mathrm{C}$ after 60 days determined an acidity level between 0.192 (Jam 1) and 0.301 (Jam 6). Maximum increase was observed in Jam $7(43.3 \%)$ followed by Jam $6(36.6 \%)$, while minimum increase was observed in Jam 1 (19.5\%) followed by Jam 3 (20.8\%). The results are in agreement with Muhammad et al. [15], which observed increased in \% acidity from $42.22 \%$ to $71.88 \%$ in apple diet jams. Formation of organic acids due to degradation of polysaccharides and breaking of chemical bonds might be the reason for this increase in acidity [16]. Under light conditions, the 
increasing of TA was more pronounced than under dark conditions, due to a higher degradation of jam components. Also, there was a higher increase of $\mathrm{TA}$ at $25^{\circ} \mathrm{C}$ than at $4^{\circ} \mathrm{C}$, suggesting that temperature of storage affects this parameter. This is also confirmed by one-way ANOVA test. Thus, it was observed that the differences on TA were not significant $(p>0.05)$ after 15 days of storage at $4^{\circ} \mathrm{C}$, but after 30 and 60 days of storage, respectively, the changes became significant for all jams samples $(p<0.05)$. Storage at $25^{\circ} \mathrm{C}$ both under dark and light conditions induced significant differences reported to the control values $(p<0.05)$. It was noticed that the changes in TA were significantly affected by the type of sweetener used in jam formulation $(p<0.05)$. In addition, two-way ANOVA shows that between storage conditions and jams formulations no significant interaction effect exists $(p>0.05)$.

\section{Effects of storage time and temperature on total soluble solids (TSS) of jams samples}

In this study, TSS of the products served as control parameter. When TSS reached 56.0-57.0 ${ }^{\circ}$ Brix, jams heating was stopped, excepting Jam 6 and Jam 7, formulated with stevia and saccharine, respectively. In the case of these jams, heating was stopped when TSS reached about $22.0^{\circ} \mathrm{Brix}$, as previously reported by [16]. The TSS is primarily represented by sugars, with acids and minerals contributing, being an important parameter for evaluating jams quality [17]. Prior storage, TSS values of lingonberry jams were: 56.2 ${ }^{\circ} \mathrm{Brix}$ (Jam 1), $56.7^{\circ} \mathrm{Brix}$ (Jam 5), $56.8^{\circ} \mathrm{Brix}$ (Jam 2 and 3), $57.0^{\circ} \mathrm{Brix}$ (Jam 4), while in the case of Jams 6 and 7, Brix values were much lower (22.0 and $21.7^{\circ} \mathrm{Brix}$, respectively). ${ }^{\circ}$ Brix values of the jams samples analyzed during 60 days are presented in Table 1.

The data obtained for TSS revealed that there was a very slight change occurred during 60 days of storage at $4^{\circ} \mathrm{C}$. Generally, all samples show an increasing in TSS during storage. Maximum increase was observed in Jam $6(3.63 \%)$, while minimum increase was observed in Jam 2 (0.176\%). These results are in agreement with the results of Muhammad et al. [15] who observed an increase in TSS of apple jams during storage. In another study Ehsan et al. [18] reported a slight increase in TSS of watermelon lemon jam from 68.6 to 68.9 during 60 days of storage. The increase in TSS contents may be explained by the solubilization of jam constituents during storage [15].

The high content of TSS might also be due to hydrolysis of polysaccharide especially pectin into simple sugar in the presence of acid during storage [16]. Moreover, increasing of TSS content was more visible in the case of samples stored at $25^{\circ} \mathrm{C}$, exposed to light conditions, than those 
stored under refrigeration. The highest mean value was recorded for Jam 6 $\left(23.0^{\circ} \mathrm{Brix}\right)$ and Jam $4\left(57.7^{\circ} \mathrm{Brix}\right)$ after 60 days of storage, both under light conditions, indicating that light and temperature influence the TSS.

Table 1. The TA and TSS values of jams samples obtained during storage.

\begin{tabular}{|c|c|c|c|c|c|c|}
\hline \multirow{2}{*}{$\begin{array}{c}\text { Time } \\
\text { (days) }\end{array}$} & \multicolumn{3}{|c|}{ Titratable acidity (\%) } & \multicolumn{3}{|c|}{ Total soluble solids ( ${ }^{\circ} \mathrm{Bx}$ ) } \\
\hline & $4^{\circ} \mathrm{C}$ & $25^{\circ} \mathrm{C}$ (dark) & $25^{\circ} \mathrm{C}$ (light) & $4^{\circ} \mathrm{C}$ & $25^{\circ} \mathrm{C}$ (dark) & $25^{\circ} \mathrm{C}$ (light) \\
\hline \multicolumn{7}{|c|}{ Jam 1} \\
\hline 0 & 0.161 & 0.161 & 0.161 & 56.2 & 56.2 & 56.2 \\
\hline 15 & 0.168 & 0.168 & 0.175 & 56.3 & 56.4 & 56.6 \\
\hline 30 & 0.189 & 0.189 & 0.192 & 56.4 & 56.8 & 56.8 \\
\hline 60 & 0.192 & 0.195 & 0.203 & 56.5 & 56.9 & 56.9 \\
\hline \multicolumn{7}{|c|}{ Jam 2} \\
\hline 0 & 0.182 & 0.182 & 0.182 & 56.8 & 56.8 & 56.8 \\
\hline 15 & 0.196 & 0.210 & 0.224 & 56.8 & 56.8 & 56.9 \\
\hline 30 & 0.245 & 0.248 & 0.315 & 56.8 & 56.8 & 57.0 \\
\hline 60 & 0.273 & 0.280 & 0.315 & 56.9 & 56.9 & 57.4 \\
\hline \multicolumn{7}{|c|}{ Jam 3} \\
\hline 0 & 0.168 & 0.168 & 0.168 & 56.9 & 56.9 & 56.9 \\
\hline 15 & 0.175 & 0.175 & 0.182 & 43.4 & 44.3 & 45.2 \\
\hline 30 & 0.182 & 0.182 & 0.196 & 43.7 & 44.6 & 44.8 \\
\hline 60 & 0.203 & 0.189 & 0.217 & 44.0 & 44.1 & 44.2 \\
\hline \multicolumn{7}{|c|}{ Jam 4} \\
\hline 0 & 0.161 & 0.161 & 0.161 & 57.0 & 57.0 & 57.0 \\
\hline 15 & 0.175 & 0.182 & 0.280 & 57.1 & 57.1 & 57.3 \\
\hline 30 & 0.189 & 0.199 & 0.343 & 57.2 & 57.2 & 57.6 \\
\hline 60 & 0.196 & 0.210 & 0.343 & 57.3 & 57.3 & 57.7 \\
\hline \multicolumn{7}{|c|}{ Jam 5} \\
\hline 0 & 0.175 & 0.175 & 0.175 & 56.7 & 56.7 & 56.7 \\
\hline 15 & 0.210 & 0.217 & 0.315 & 56.8 & 56.8 & 56.8 \\
\hline 30 & 0.224 & 0.238 & 0.332 & 56.9 & 56.9 & 56.9 \\
\hline 60 & 0.252 & 0.273 & 0.364 & 56.9 & 57.0 & 57.2 \\
\hline \multicolumn{7}{|c|}{ Jam 6} \\
\hline 0 & 0.210 & 0.210 & 0.210 & 22.0 & 22.0 & 22.0 \\
\hline 15 & 0.280 & 0.311 & 0.343 & 23.2 & 23.4 & 23.4 \\
\hline 30 & 0.287 & 0.318 & 0.350 & 22.8 & 23.0 & 22.9 \\
\hline 60 & 0.301 & 0.343 & 0.385 & 22.9 & 22.9 & 23.0 \\
\hline \multicolumn{7}{|c|}{ Jam 7} \\
\hline 0 & 0.210 & 0.210 & 0.210 & 21.7 & 21.7 & 21.7 \\
\hline 15 & 0.273 & 0.273 & 0.315 & 21.7 & 21.7 & 22.0 \\
\hline 30 & 0.294 & 0.308 & 0.322 & 21.9 & 21.9 & 22.0 \\
\hline 60 & 0.301 & 0.308 & 0.336 & 22.1 & 22.0 & 22.0 \\
\hline
\end{tabular}


The samples stored at $25^{\circ} \mathrm{C}$ at the dark followed a similar trend as samples stored under refrigeration. The results of statistical processing by one-way ANOVA test revealed that during jam storage, the differences reported to the control were quantified as non-significant $(p>0.05)$, regardless of temperature storage. However, it could be noticed that the changes in TSS content was affected by jam's formulation $(p<0.05)$. Also, two-way ANOVA show that no significant interaction effect between storage conditions and formulation is observed $(p>0.05)$.

\section{Sensory evaluation of jams samples}

The sensory profile of the lingonberry jams was evaluated in terms of color, taste, texture, spreadability and overall acceptability. The sensory evaluation indicated that the majority of jams were acceptable to the consumers after 180 days of storage at $4{ }^{\circ} \mathrm{C}$ (Table 2). Result showed that Jam 5 recorded the best sensory evaluations, except for the color. Color is an important sensory attribute on which the consumer preference depends. Color acceptability was highest for Jam 6 and Jam 7 (8.80) while Jam 3 gave lowest (5) means value color acceptability.

Table 2. Sensory parameters of the jams samples.

\begin{tabular}{|l|c|c|c|c|c|c|c|}
\hline Jam Parameter & 1 & 2 & 3 & 4 & 5 & 6 & 7 \\
\hline Color & 8.60 & 8.00 & 5.00 & 8.60 & 6.20 & 8.80 & 8.80 \\
\hline Taste & 7.80 & 7.00 & 4.20 & 7.20 & 8.80 & 8.00 & 6.80 \\
\hline Texture & 8.80 & 7.40 & 4.20 & 8.40 & 8.80 & 8.00 & 8.00 \\
\hline Spreadability & 8.80 & 6.40 & 4.00 & 8.60 & 8.80 & 8.40 & 8.20 \\
\hline Overall Acceptability & 8.20 & 7.60 & 3.80 & 8.20 & 8.80 & 8.40 & 8.00 \\
\hline
\end{tabular}

For taste, the maximum mean value was obtained in the case of Jam 5 (8.80), followed by Jam 6 (8) and Jam 1 (7.80). The high value of Jam 5 could be as a result of the presence of coconut sugar, that has a distinctive aroma, slightly acidic and smelling caramel. Texture ranged from 4.20 to 8.80 with Jam 3 having the lowest value while Jam 1 and Jam 5 had the highest. The low texture of Jam 3 could be attributed to the sweetener agent that crystallized during storage. Decrease in texture quality might be also due to hydrolysis of pectin contained in fruits and sweeteners during storage. These results are in agreement with Abolila et al. [1] who observed decreasing trend in texture of low calorie orange jam during storage. Concerning spreadability, Jam 3 and Jam 2 obtained the lowest scores (4 and 6.40, respectively). 
Overall acceptability of the jam samples ranged from $3.80-8.80$ and Jam 5 and 6 were rated most preferred while Jam 3 was least preferred. The sensory scores indicate that lingonberry jam samples were highly acceptable by the consumers except for the jam containing erythritol (Jam 3), which had the least preference scores compare to other jams. Overall acceptability is beyond 7.60 on a 9-point hedonic scales in the case of all jams, excepting Jam 3 , revealing that they were equally acceptable by the panelists. The high sensory values of these jams could be due to the color and flavor of lingonberry fruits that are transferred to the final products on processing. Additionally, jam prepared with stevia (Jam 6) was evaluated as being better than jam prepared with sucrose. Since stevia extracts possess antioxidant, antimicrobial and antifungal activity $[10,11]$, jams formulated with stevia could be commercialized as functional products. Moreover, although the bitterness level for the Jam 7 was expected to be very high, due to the saccharine content, it seems that the combination of this sweetener with lingonberry fruits reduced bitterness of jam, being preferred by the panelists, especially for its color and spreadability.

\section{CONCLUSIONS}

The results of our study supplies information regarding the replacement of sucrose from lingonberry jams with other sweeteners (natural and synthetic) and describes the sensorial stability of these jams during storage. The use of several sweeteners in the manufacture of lingonberry jam was shown to be satisfactory, resulting in a product with jam characteristics, taste and texture similar to conventional jam with sucrose, excepting jam prepared with erythritol. Storage at $4^{\circ} \mathrm{C}$ induced changes on TA and TSS parameters of lingonberry jams, but not as important as a storage at $25^{\circ} \mathrm{C}$, indicating a better preservation of jams under refrigeration, regardless sweetener agent. Sugar formulation affected sensorial parameters of jams. Jams formulated with stevia and saccharine maintained the best color during storage, while jam formulated with coconut sugar obtained the lowest scores regarding color. The formulations containing sucrose or coconut sugar were the easiest to spread and resulted in the highest maintenance of texture. Coconut sugar led to the best scores for taste, mainly due to its distinctive flavor. Although jams prepared with stevia and saccharine did not reach the same concentration of soluble solids as jams made with sucrose, they were stable over the storage period considered. Moreover, the use of natural sweetener stevia in the manufacture of lingonberry jam resulted in a product with a higher overall acceptability than jam prepared with sucrose, this study being useful for diabetics or even for weight maintaining persons. 


\section{EXPERIMENTAL SECTION}

\section{Chemicals and reagents}

All chemicals and reagents used in this study were of analytical grade and were purchased from Merck, Germany. Distilled water was used for solutions preparation.

\section{Fruit material and jams preparation}

Fresh lingonberries (Vaccinium vitis-idaea L.) were harvested from Apuseni Mountains, Romania, in August, 2019. Lingonberry jams were prepared in the laboratory, according to a traditional procedure. Basic formulation used for of each type of jam is presented in Table 3. White sugar was purchased from a local market in Cluj-Napoca, while all the other sweeteners used in this study were purchased from a health food store. The ingredients were heated at low temperature $\left(50{ }^{\circ} \mathrm{C}\right)$, which was monitored during the entire process. Boiling process was stopped when TSS (total soluble solids) reached $56-57^{\circ}$ Brix, excepting Jam 6 and Jam 7 , formulated with stevia and saccharine, respectively. In the case of these jams, heating was stopped when TSS reached about $22^{\circ}$ Brix, as previously reported by [16]. Each jam was packed into glass jars with screw caps, without being pasteurized. The jams were stored at $5^{\circ} \mathrm{C}$, at $25^{\circ} \mathrm{C}$ (under light conditions) and at $25^{\circ} \mathrm{C}$ (under darkness conditions). Samples were analyzed immediately and after 15,30 and 60 of storage.

Table 3. Jams formulation

\begin{tabular}{|c|c|c|}
\hline & \begin{tabular}{|} 
Lingonberry fruits \\
$(\mathrm{g})$
\end{tabular} & $\begin{array}{l}\text { Sweetener } \\
\text { (g) }\end{array}$ \\
\hline Jam 1 (with white sugar) & 100 & 50.0 \\
\hline Jam 2 (with fructose) & 100 & 29.4 \\
\hline Jam 3 (with erythritol) & 100 & 77.0 \\
\hline Jam 4 (with brown sugar) & 100 & 50.0 \\
\hline Jam 5 (with coconut sugar) & 100 & 50.0 \\
\hline Jam 6 (with stevia) & 100 & 0.180 \\
\hline Jam 7 (with saccharine) & 100 & 0.180 \\
\hline
\end{tabular}




\section{Titratable Acidity (TA) determination}

Titratable acidity (TA) was determined as previously described by Awolu et al. [19]. About $1 \mathrm{~g}$ of each sample was weighed and put into $50 \mathrm{ml}$ centrifuge tube. Distilled water was added the centrifuge tube, mixed with the sample and filtered. About $1 \mathrm{ml}$ aliquot of the filtered solution was taken and diluted with $10 \mathrm{ml}$ of distilled water. Aliquotes of diluted samples $(10 \mathrm{ml})$ were titrated with volumetric solution of $0.1 \mathrm{~N} \mathrm{NaOH}$ and the volume of $\mathrm{NaOH}$ was converted to percentages of citric acid, using equation (1):

$$
\% \text { citric acid }=\mathrm{V}_{\mathrm{NaOH}} / \mathrm{V}_{\text {sample }} \times 0.007 \times 100
$$

where $\mathrm{V}_{\mathrm{NaOH}}$-volume of alkali used in titration and $\mathrm{V}_{\text {sample-volume of the }}$ sample titrated $(10 \mathrm{ml})$.

\section{Total Soluble Solids (TSS) determination}

Total concentrations of soluble solids were determined using a Bellingham + Stanley refractometer (Bellingham + Stanley Ltd., Kent, UK) and expressed as degrees Brix ( $\left.{ }^{\circ} \mathrm{Brix}\right)$. The instrument prism was covered with about 1-2 drops of cooled lingonberry jam and soluble solids value was directly recorded from the digital reading display at ambient temperature.

\section{Sensory analysis}

All evaluation sessions were held in an analytical laboratory from our university and were conducted by an untrained panel consisting of 5 students with 24 years mean age. Color, taste, texture, spreadability and overall acceptability were evaluated according to the hedonic scale of nine points ( 9 $=$ like extremely to $1=$ dislike extremely) as reported by [17]. Jam samples were tempered for $30 \mathrm{~min}$ at room temperature $\left(22 \pm 2{ }^{\circ} \mathrm{C}\right)$ and drinking water was provided after each sample testing.

\section{Statistical analysis}

The experimental results were subjected to statistical analysis using Microsoft Excel. One-way analysis of variance (ANOVA) was used to determine significant differences between values. The significance level was defined as $p<0.05$ for $95 \%$ probability. Two-way ANOVA was used to identify the interdependence between factors. 


\section{ACKNOWLEDGMENTS}

The authors would like to thank the project POCU/380/6/13/123886"Entrepreneurship for innovation through doctoral and postdoctoral research" for the financial support given to this investigation.

\section{REFERENCES}

1. R. M. Abolila; H. Barakat; H. A. El-Tanahy; H. A. El-Mansy; Food Sci. Nutr., 2015, 6, 1229-1244.

2. V. R. Souza; P.A. Pereira; A. C. M. Pinheiro; H. Bolini; S.V. Borges; F. Queiroz; Int. J. Food Sci. Technol., 2013, 48, 1541-1548.

3. C. Mane; M. Loonis; C. Juhel; C. Dufour; C. Malien-Aubert; J. Agric. Food Chem, 2011, 59, 3330-3339.

4. M. Toivanen; S. Huttunen; S. Lapinjoki; C. Tikkanen-Kaukanen; Phytother Res., 2011, 25, 828-832.

5. A. Vilela; S. Matos; A. S. Abraão; A. M. Lemos; F. M. Nunes; J. Food Process, 2015, 2015, 1-14.

6. S. Basu; U. S. Shivhare; J. Food Eng., 2010, 100 (2), 357-365.

7. Y. Akanksha; G. Gulia; Y. Bhuvnesh; Int. J. Sci. Eng., 2020, 9(1), 4079-4083.

8. M. Belovik; A. Torbika; I. P. Lijakovic; J. Mastilovik; Food Chem., 2017, 237, 1226-1233.

9. M. Grembecka; Eur Food Res Technol., 2015, 241, 1-14.

10. R. Lemus-Mondaca; A. Vega-Gálvez; L. Zura-Bravo; K. Ah-Hen; Food Chem., 2012, 132, 1121-1132.

11. M. R. Mora; R. Dando; Compr Rev Food Sci Food Saf., 2021, 1-30.

12. G. E. DuBois; I. Prakash; Annu Rev Food Sci Technol., 2012, 3, 353-380.

13. S. Basu; U. S. Shivhare; T. V. Singh; J. Food Eng, 2013, 114, 465-476.

14. K. C. Foletto; B. A. Melo Batista; A. M. Neves; F. deMatos Feijó; C.R. Ballard; M. F. Marques Ribeiro; M. C. Bertoluci; Appetite, 2016, 96, 604-610.

15. A. Muhammad; Y. Durrani; A. Zeb; M. Ayub; J. Ullah; Sarhad J. Agric.,2008, 24 (3), 461-467.

16. R. Sutwal; J. Dhankhar; P. Kind; R. Mehla; Int. J. Curr. Res., 2019, 11(4), 9-16.

17. N. Touati; M. P. Tarazona-Díaz; E. Aguayo; H. Louaileche; Food Chem., 2014, 145, 23-27.

18. E.B. Ehsan; Z.P. Naeem; A. Ghafoor; M.S. Bahtti; Pak. J. Food Sci., 2002, 12 (3-4), 21-24.

19. O. O. Awolu; G. O. Okedele; M. E. Ojewumi; G. F. Oseyemi; J. Int. J. Biotechnol. Food Sci., 2018, 3(1), 7-14. 